\title{
A Scoping Review Exploring Psychological Resilience in Relation to Various Biomolecular variables
}

\author{
Ulrika Axelsson ${ }^{1 \# *}$, Johanna Månsson ${ }^{2,3 \#}$, Per Johnsson ${ }^{3}$, Carl AK Borrebaeck ${ }^{1}$, Lisa Rydén ${ }^{4}$, Patrik \\ Edén $^{5}$ and Ingalill Rahm Hallberg ${ }^{6}$ \\ ${ }^{1}$ Department of Immunotechnology and CREATE Health Translational Cancer Center, Sweden \\ ${ }^{2}$ Department of Clinical Sciences, Lund, Division of Paediatrics, Sweden
}

${ }^{3}$ Department of Psychology, Sweden

${ }^{4}$ Department of Clinical Sciences, Lund, Division of Surgery and Department of Surgery, Sweden

${ }^{5}$ Computational Biology and Biological Physics, Department of Astronomy and Theoretical Physics, Sweden

${ }^{6}$ Department of Health Sciences, Sweden

"Those authors contributed equal

*Corresponding author: Ulrika Axelsson, Department of Immunotechnology and CREATE Health Translational Cancer Center, Lund University, Medicon Village, 22381 Lund, Sweden

\section{ARTICLE INFO}

Received: 幽 December 02, 2019

Published: December 11, 2019

Citation: Ulrika Axelsson, Johanna Månsson, Per Johnsson, Carl AK Borrebaeck, Lisa Rydén, et al. A Scoping Review Exploring Psychological Resilience in Relation to Various Biomolecular variables. Biomed J Sci \& Tech Res 23(5)-2019. BJSTR. MS.ID.003960.

Keywords: Psychological Resilience; Biomolecular Variables; Scoping Review; Cancer

\section{ABSTRACT}

Objective: This scoping review aimed to explore the research investigating the relationship between psychological resilience and biomolecular variables, especially those related to cancer. The goal was to obtain a knowledge base that could advise further studies of the interaction between these aspects.

Method: A systematic literature search of psychological resilience and various biomarkers initially resulted in 252 possible studies, of which 63 were read in full and 16 fulfilled the inclusion criteria.

Result: The most used measure for psychological resilience was the CD-RISC 25 or 10 , and the most common biomarkers were the promoter region of the serotonin transporter gene and inflammatory markers. However, the traumatic situations explored varied and very few related to the context of cancer.

Conclusion: It seems worthwhile to explore psychological measures in relation to biomarkers in cancer. Indications of such relationships were detected, although the studies conducted thus far were few. Consequently, a more consistent approach is needed to further strengthen the knowledge base.

\section{Background}

Psychological resilience has been studied by researchers from a wide range of disciplines and scientific arenas. Early studies focused primarily on adaptive behaviors in children facing adversities such as maltreatment, parental mental illness, and poverty. Researchers found that some individuals have a remarkable ability to develop normally despite these difficulties. Psychological resilience was considered reflecting an extraordinary inner strength and an ability to make the most of a "bad hand". Personal traits, or individual assets, such as intellectual functioning and positive self-esteem,

were of main interest to the field. As the research has increased, the understanding of resilience as a complex process of biological, psychological, social, cultural factors has continuously developed [1]. Although most agree that (positive) adaptation and adversity constitute the core elements of psychological resilience, there is considerable variety in the proposed definitions within the literature (see, for example, reviews by [2,3]). The types of adversity included in studies of psychological resilience range from comparably mild difficulties and daily hassles (e.g. work stress) to extensive stress and severe trauma (e.g. bereavement or life-threatening events). 
Furthermore, the definitions of adaptation in the resilience literature may vary, depending, for example, on the nature of the adversity. Adaptation to severe adversity may be defined in terms of an absence of psychiatric diagnoses, whereas adaptation to mild adversity could be defined as maintaining psychological health and achieving goals [2,4]. Personal factors such as self-efficacy, spirituality, and adaptability, as well as systemic microenvironmental (e.g. social support from family and friends) and macroenvironmental (e.g. health care services) factors, may increase psychological resilience [3]. There has also been a growing interest in the interaction between biological and genetic factors and psychological resilience. Genetically informative research is important for understanding the processes underlying individual variability in response to stress and, consequently, for providing a scientific basis for interventions to support psychological resilience [5]. Genetic research has enhanced the knowledge of resilience through studies of complex gene $\mathrm{x}$ environment ( $\mathrm{G} \times \mathrm{E}$ ) interaction processes, in which the health effects that arise from exposure to risk factors are moderated by certain genetic variations.

For example, studies of the genetic etiology of psychopathology have identified individuals with the low-activity allele of the monoamine oxidase-A (MAO-A) gene as being at increased risk of developing behavioral problems if they have experienced childhood maltreatment. However, without maltreatment, this genetic variation does not exacerbate the risk of behavioral problems $[6,7]$. There are repeated reports that having two copies of the 5-hydroxytryptamine-linked polymorphic region polymorphism (5-HTTLPR) short allele (S) seems to be linked to emotional problems. 5-HTTLPR is the promoter region of the serotonin transporter (5-HTT) gene. The 5-HTTLPR genetic variation increases vulnerability to depression and anxiety in contexts of high-stress childhood environments; otherwise it does not. Studies have shown that individuals carrying 5-HTTLPR S alleles function better than carriers of long alleles (L) when not exposed to stressors (see for example ref. Kim-Cohen, et al. [8], Belsky and Pluess [9]). Hence, early life experiences may cause lasting epigenetic changes that modify gene expression which, subsequently, can affect the individual response to environmental stressors during adulthood. In addition, these genetic variations increase plasticity and susceptibility to environmental effects [9]. Kim-Cohen and Gold [7] argue that these genes might predict psychological resilience to adversity, as well as predict psychopathology.

In medicine, there is a growing understanding of the role of psychosocial factors in disease incidence and progression. Cal and coworkers [10] identified negative correlations between psychological resilience and symptom activity for diseases, such as diabetes and Parkinson's disease. High resilience was associated with health-promoting behaviors and treatment adherence. Additionally, some studies have described a link between stressful experiences and later cancer initiation [11]. Cancer patients are at a heightened risk for depression, which are, in turn, associated with poorer survival potentially due to an increased metastatic activity. Pinquart and Duberstein $[12,13]$ describe the importance of social support in cancer survival. Lutgendorf and Andersen [14] outline how psychological and behavioral processes can affect biological variables involved in tumor progression. Studies show that stress peaks at the time of diagnosis (both initial diagnosis and recurrence). If stress levels are continuously high, there is a heightened risk for diminished cellular immunity and poorer cellular response to specific antigens [14]. Interestingly, Creed and coworkers [15] showed that stressed induced neural signaling affected the migration pattern of tumor cells, indicating an increased metastasizing ability. It is therefore obvious that the ability to regain psychological stability when facing such a risk is crucial. Clinically, it is important to identify those least able to manage the stressor of cancer and who, thus, are at increased risk of having a poor outcome and lower quality of life. Lutgendorf and Andersen [14] identify the need for a more nuanced understanding of the role of psychosocial factors during the cancer trajectory. Thus far, little research has been performed combining measures of psychological resilience and biomolecular parameters. Research questions regarding stress reactions, resilience and coping with treatment are generally addressed within psychology, health care and nursing research domains. However, biomolecular research seldom includes psychological parameters, such as the subjective capacity to manage stressors. Thus, the two perspectives seem to be investigated in different research areas and bringing them together may result in a breakthrough in research and treatment.

\section{Aim}

The main objective of this report is to provide a scoping review of the relationship between psychological resilience and biomolecular processes, particularly the relationship in a cancer context.

\section{Methods}

The review procedure used in this work is based on the methodological framework described by Arksey and O'Malley [16]. Scoping reviews are suitable when the current knowledge base is sparse and thus it is expected to need a broad approach. The procedure occurs in five stages:

1) Identifying the research question

2) Identifying relevant studies

3) Study selection

4) Charting the data

5) Summarizing and reporting the results

\section{Identifying the Research Question}

Our primary research question was "What is known in the existing scientific literature about how and if psychological resilience can be coupled to biomolecular parameters in the context of cancer representing a traumatic life experience? 


\section{Identifying Relevant Studies}

Primary searches were performed, using electronic databases including PubMed, PsycInfo, MEDLINE, CINAHL Complete, Social Sciences Citation Index, Scopus, and Science Direct between August 2015 and January 2016. Based on the aim and the primary research question of the present study, the following search terms were used and combined:

\section{Psychological resilience}

and

2. Biomolecular markers/biomarkers

3. Protein (proteomics, proteome)

4. Gene (genomics, genome)

5. Chromatine (chromatine changes)

6. DNA methylation

7. MicroRNA (miRNA, mRNA)

8. Immune response

and

9. Cancer

10. Neoplasm

Primary searches, using the strategy presented above, identified only few references. Data were found to be too scarce to synthesize to perform a systematic summary of the research topic. The research group, therefore, decided to broaden the scope of the research question to: What is known in the existing scientific literature about the connection between psychological resilience and biomolecular variables? The same search terms were used, excluding "Cancer" and "Neoplasm". There was no limit applied regarding publication date. Thesaurus examinations indicated that psychological resilience was introduced as a scientific term in 2003, replacing the discontinued term hardiness. In databases, such as PsycInfo and PsycExtra, hardiness was then removed from all records containing it and replaced with psychological resilience. In addition to database searches, a few more references were identified via hand searches of reference lists or via conference abstracts.

\section{Study Selection}

Articles were included in the review if they met the following criteria:

1. Published in a peer-reviewed journal

2. Described research methods

3. Included primary research

4. Published in full text
5. Addressed the potential relationship between psychological resilience and biomarkers

6. Included human subjects

7. Written in the English language

Articles were excluded from the review if they met any of the following criteria:

1. Review study

2. Not in English

3. Did not investigate the relationship between psychological resilience and biomarkers

4. Focused exclusively on specific clinical cohorts, such as mental illness

5. Included animal subjects only

An EndNote library was used for collecting, managing and keeping track of records. During the reference selection process, abstracts and full text articles were independently assessed by a group of experts representing different scientific disciplines (computational biology, biological physics, health-promoting interventions, immunology, molecular biology, oncology, and psychology). Regular meetings facilitated agreement between different assessors. The first decisions were based on abstracts, evaluated against the predetermined inclusion and exclusion criteria. For studies that appeared suitable for inclusion, the full text versions were obtained and evaluated, and the final decision was made (Figure 1). After duplicates were removed, the abstracts of 252 references were screened. The rationale for exclusion was primarily based on study subjects (animal subjects) and study cohorts (specific clinical cohorts such as patients suffering from diabetes, severe psychiatric illnesses or various addictions). Sixtythree references were selected for full-text readings (Appendix 1), and 47 were excluded for the following reasons:

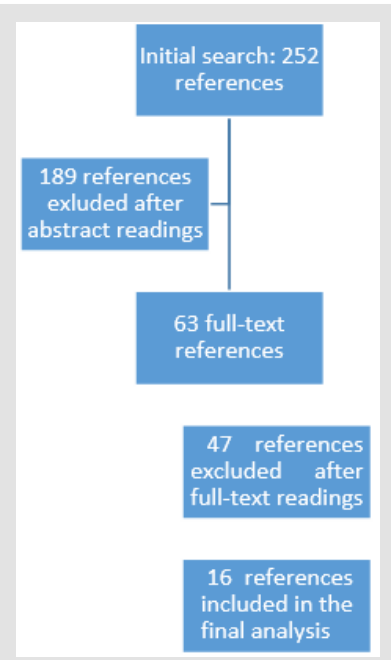

Figure 1: Selection process for included articles. 
1. Twenty-one did not present primary research but were reviews, editorials, articles with theoretical approaches or book chapters.

2. Eleven did not include psychological resilience as a variable but focused on post-traumatic responses or chronic stress or maltreatment.

3. Ten focused on specific clinical cohorts.

4. Two did not include interpretable biological markers.

5. Two did not focus on psychological resilience but on secure attachment or traits such as sociability.

6. One did not include a complete methodological description.

\section{Results}

\section{Charting the Data}

Key pieces of information from the included studies were extracted and charted to facilitate data comparison (Appendix 2). Out of the 252 references included for abstract review, 16 references are included in this scoping review. These 16 references investigate the influence of psychological parameters, such as resilience, depression and stress, in persons exposed to a variety of potentially traumatic or disturbing events and if and how those psychological parameters are coupled to biomolecular markers. Only three references investigated a cancer indication: ovarian cancer $[17,18]$ and radical prostatectomy or radiation therapy [19]. Thus, references that investigate potential traumas

(i) Childhood adversity [20,21],

(ii) War zone stressors [22,23],

(iii) High-impact trauma [24,25]

(iv) Stress (acute and chronic) [26-28] were included, as well.

(v) Additionally, two studies looking at the role of psychological resilience in successful aging and if it is reflected in biomolecular markers $[29,30]$

and

(vi) Two cross-sectional studies investigating psychological resilience and biomolecular markers in nonclinical community populations [31,32] were included.

Table 1: Summary of biomolecular markers analyzed in the included articles.

\begin{tabular}{|c|c|c|}
\hline Biomarker identified & Biomarker (short) & References \\
\hline Matrix metalloproteinases - 2 & MMP-2 & Lutgendorf et al. [18] \\
\hline Matrix metalloproteinases - 9 & MMP-9 & Lutgendorf et al. [18] \\
\hline Vascular Endothelial Growth Factor & VEGF & Lutgendorf et al. [18] \\
\hline Norepinephrine & $\mathrm{NE}$ & Lutgendorf et al. [18] \\
\hline Tumour Norepinephrine & Tumour NE & Davis et al. [17] \\
\hline Cortisol & - & $\begin{array}{l}\text { Lutgendorf et al. [18] Aschbacher et al. [28] } \\
\text { Petros et al. [31] }\end{array}$ \\
\hline Tumor Necrosis Factor Receptor 2 & TNF-RII & Hoyt et al. [19] \\
\hline C-reactive protein & CRP & Hoyt et al. [19] Gill et al. [25] \\
\hline Interleukin 6 & IL-6 & $\begin{array}{c}\text { Cole et al. [26] Gill et al. [25] Hoyt et al. [19] } \\
\text { Sandvik et al. [27] }\end{array}$ \\
\hline $\begin{array}{l}\text { 5-hydroxytryptamine- linked polymorphic region polymorphism } \\
\text { short allele /long allele polymorphism }\end{array}$ & $\begin{array}{l}\text { 5-HTTLPR short/long poly- } \\
\text { morphism }\end{array}$ & $\begin{array}{c}\text { Carli et al. [20] } 0 \text { Hara et al. [30] Stein et al. [32] } \\
\text { Telch et al. [23] }\end{array}$ \\
\hline Buccal Epithelial Cells & & Das et al. [21] \\
\hline $\begin{array}{l}\text { he Dopamine receptor D4 (DRD4) exon III variable number tan- } \\
\text { dem repeat (VNTR) genotype }\end{array}$ & DRD4-exIII-VNTR genotype & Das et al. [21] \\
\hline Interleukin 12 & IL-12 & Sandvik et al. [27] \\
\hline Interleukin 4 & IL-4 & Sandvik et al. [27] \\
\hline Interleukin 10 & IL-10 & Sandvik et al. [27] \\
\hline Neuro-peptide-Y & NPY & Sandvik et al. [27] \\
\hline rapid eye movement sleeps periods & REM sleeps periods & Cowdin et al. [24] \\
\hline Lipid concentrations & & Gill et al. [25] \\
\hline Single Nucleotide Polymorphism & SNP's & Rana et al. [29] \\
\hline Dehydroepiandrosteron sulfat & DHEA-S & Petros et al. [31] \\
\hline 8-Hydroxyguanosine & & Aschbacher et al. [28] \\
\hline 8-hydroxy-2'- deoxygyanosine & 8-OHdG & Aschbacher et al. [28] \\
\hline 8-iso-prostaglandin & 8-iso-PG & Aschbacher et al. [28] \\
\hline Right hemisphere prefrontal theta power during REM sleep & & Cowdin et al. [24] \\
\hline
\end{tabular}




\section{Summarizing and Reporting the Results}

A range of biomolecular markers and bio-behavioral conditions were analyzed in the included articles (Table 1). Regardless of the type of trauma, only a few biomolecular markers were investigated in relation to psychologic resilience.

Promoter Region of the Serotonin Transporter Gene: Genetic variants of the promoter region (5-HTTLPR) of the serotonin transporter (5-HTT) gene were investigated in five of the studies included [20,22,23,30,32]. Compared to individuals who were homozygous for the L allele, carrying one or two copies of the $\mathrm{S}$ allele of the promotor gene was found to be associated with, (i) more symptoms of Posttraumatic Stress Disease PTSD and depression in soldiers exposed to war zone stressors [23], (ii) worse cognition and self-reported ratings of successful aging [30], and (iii) lower psychological resilience in college students [32]. Interestingly, Graham et al. [22] showed that carrying the L allele was associated with lower resilience and more perceived limitations related to community participation in war veterans. Graham et al. and Carli et al. [20,22] showed that men that had been subjected to severe childhood adversities and that had the $\mathrm{L}$ allele had lower psychological resilience and higher depressive severity than men with homozygous S/S copies of the gene. In addition, there was no correlation between carrying the $S$ gene and psychological resilience in older adults (> 55 years) [30].

Inflammatory Markers: In four studies, inflammatory and anti-inflammatory markers were shown to correlate with biobehavioral conditions $[18,19,25,27]$,

(i) Individuals with high social support had lower levels of the inflammatory marker matrix metalloproteinase-9 (MMP-9) in tumor-associated macrophages (TAMs) [18];

(ii) Individuals who were more depressed and reported higher levels of stress had higher levels of MMP-9 expressing TAMs [18];

(iii) Lower levels of the inflammatory markers IL-6 and CRP were correlated with higher level of health-related quality of life (HRQOL) [25];

(iv) Lower levels of the inflammatory markers IL-6, sTNFRII and CRP were correlated with higher emotion processing ability [19]; and

(v) Lower levels of the inflammatory marker IL-12 and increased levels of the anti-inflammatory markers IL-4 and
IL-10 were correlated with individuals who were more stress reactive [27]. Combined, these results suggest an association between a more balanced psychological profile and lower levels of inflammatory markers, however the study by Sandvik et al.

[27] showed the opposite effect.

Methods used to Analyze the Psychological Parameters: The majority of the included articles used self-report questionnaires (Table 2). Seven studies [20-22,29-32] used the Connor-Davidson Resilience Scale (CD-RISC), either the 25- or the 10-item version, to similarly operationalize psychological resilience. The CDRISC conceptualizes psychological resilience as a multifactorial construct including feelings of personal competence, trust in one's instincts, positive acceptance of change, and spiritual influences [33]. One study [27] used a local adaptation of the Dispositional Resilience Scale 15-item version (DRS-15) - conceptualizing psychological resilience (expressed as hardiness) as the ability to use adaptive strategies to manage difficulties, solve problems, make decisions, and set goals during stressful events [34]. Six studies $[17,19,25,29,30,31]$ examined qualities or strategies associated with psychological resilience, such as coping strategies, self-efficacy, well-being, generalized optimism, and access to social support, and two studies included personality measures [21,32].

Eleven studies included measures of the expected effect of psychological resilience under certain circumstances. Self-reports or interviews investigating mental disorders (anxiety, depression, and negative affect) are examples of methods used, based on the assumption that high levels of psychological distress in highly stressful situations may indicate low psychological resilience. Three studies investigated the presence of Posttraumatic Stress Disease PTSD symptoms either by self-report [22,23] or by clinical interview [24]. Resilience was then defined as a lack of PTSD symptoms after trauma exposure. In contrast, self-reports of optimism, selfefficacy, or well-being despite stressful experiences (e.g., being diagnosed with cancer) indicate high resilience. Ten articles included measures of hardships [18,20-23,25,28,30-32]. Four articles used retrospective self-reports to assess negative childhood experiences due to their known impact on adult psychopathology and resilience $[20,21,31,32]$, three studies measured present life changes $[18,25,28]$, and three studies measured experiences of specific stressors, such as concussion, combat or returning home after military service $[22,23,30]$ (Table 2 ). Two studies investigated neurophysiological responses to stress or trauma [24,28].

Table 2: Methods used to Analyze Psychological Parameters.

\begin{tabular}{|c|c|c|}
\hline Method to Measure Psychological Resilience & $\begin{array}{c}\text { Short } \\
\text { Name }\end{array}$ & References \\
\hline Connor-Davidson Resilience Scale, 10-item version & CD-RISC & O Hara et al. [30] Stein et al. [32] \\
\hline Connor-Davidson Resilience Scale, 25-item version & CD-RISC & $\begin{array}{r}\text { Carli et al. [20] Das et al. [21] Graham et al. [22] Petros et al. [31] } \\
\text { Rana et al. [29] }\end{array}$ \\
\hline Norwegian Adaptation of Dispositional Resilience Scale & DRS-15-R & Sandvik et al. [27] \\
\hline $\begin{array}{c}\text { Methods to measure qualities associated with psychological } \\
\text { resilience }\end{array}$ & \\
\hline
\end{tabular}




\begin{tabular}{|c|c|c|}
\hline Emotional approach coping & EAC & Hoyt et al. [19] \\
\hline Generalized Self-Efficacy Scale & GES & Petros et al. [31] \\
\hline Life Orientation Test - Revised & LOT-R & Rana et al. [29] Petros et al. [31] \\
\hline The Ryff Psychological Well-Being Scales & PWBS & Davis et al. [17] \\
\hline The MOS 36-item short-form health survey & SF-36 & Gill et al. [25] \\
\hline The MOS 12-item short-form health survey & SF-12 & O Hara et al. [30] \\
\hline \multicolumn{3}{|l|}{ Personality measures } \\
\hline NEO-Personality Inventory-Revised & NEO-PI-R & Stein et al. [32] \\
\hline Behavioral Inhibition System & BIS & Das et al. [21] \\
\hline Behavioral Activation System & BAS & Das et al. [21] \\
\hline \multicolumn{3}{|l|}{ Methods to measure the effect of psychological resilience } \\
\hline \multicolumn{3}{|l|}{ Mental disorders } \\
\hline \multicolumn{3}{|l|}{ Affective response } \\
\hline Positive and Negative Affect Schedule & PANAS & Aschbacher et al. [28] \\
\hline The Profile of Mood States Short form & POMS-SF & Davis et al. [17] Lutgendorf et al. [18] \\
\hline \multicolumn{3}{|l|}{ Anxiety } \\
\hline State-Trait Anxiety Inventory & $\begin{array}{l}\text { STAI- } \\
\text { Form-Y }\end{array}$ & Petros et al. [31] \\
\hline \multicolumn{3}{|l|}{ Depression } \\
\hline Beck Depression Inventory & $\mathrm{BDI}$ & Stein et al. [32] \\
\hline Hamilton Depression Rating Scale & HDRS & Carli et al. [20] \\
\hline $\begin{array}{l}\text { Center for Epidemiological Studies-Depression scale, } 20 \text {-item } \\
\text { version }\end{array}$ & CES-D & $\begin{array}{l}\text { Davis et al. [17] Graham et al. [22] Lutgendorf et al. [18] Rana et al. } \\
\text { [29] } \\
\text { Telch et al. [23] }\end{array}$ \\
\hline \multicolumn{3}{|l|}{ Trauma responses } \\
\hline Post-Traumatic Symptom Inventory - Short & PCL-S & Telch et al. [23] \\
\hline Post-Traumatic Symptom Inventory - Civilian & PCL-C & Graham et al. [22] \\
\hline The Clinician Administered PTSD Scale & CAPS & Cowdin et al. [24] \\
\hline The Impact of Event Scale & IES & Davis et al. [17] Lutgendorf et al. [18] \\
\hline \multicolumn{3}{|l|}{ Methods to measure stressors } \\
\hline \multicolumn{3}{|l|}{ Childhood stressors } \\
\hline Childhood Adversity & $\mathrm{CA}$ & Das et al. [21] \\
\hline Childhood Trauma Questionnaire & CTQ & Carli et al. [20], Stein et al. [32] \\
\hline Early Life Stressor Inventory & ELSI & Petros et al. [31] \\
\hline \multicolumn{3}{|l|}{ Present stressors } \\
\hline The Life Experience Survey & LES & Lutgendorf et al. [18] \\
\hline Life Events Checklist & & Gill et al. [25] \\
\hline The Perceived Stress Scale & PSS & Aschbacher et al. [28], Lutgendorf et al. [18] \\
\hline \multicolumn{3}{|l|}{ Specific stressors } \\
\hline Neurobehavioral Symptom Inventory & NBSI & Graham et al. [22] \\
\hline Cognitive Assessment Screening Test & CAST & O'Hara et al. [30] \\
\hline Traumatic Brain Injury Diagnosis (Questionnaire) & TBID & Graham et al. [22] \\
\hline Combat Experience Log & CEL & Telch et al. [23] \\
\hline Community Reintegration of Service Members Instrument & CRIS & Graham et al. [22] \\
\hline \multicolumn{3}{|l|}{ Neuro-physiological Measures } \\
\hline The Trier Social Stress Task & TSST & Aschbacher et al. [28] \\
\hline
\end{tabular}


Psychological Resilience and Biomolecular Markers Related to Specific Types of Trauma:

A. Cancer: Lutgendorf et al. [18] showed that ovarian cancer patients who are more depressed and who report higher levels of stress have higher levels of MMP-9 expressing TAMs. They also showed that patients who perceived that they had access to supportive social relationships had lower levels of MMP-9 and vascular endothelial growth factor (VEGF) expressed in tumor cells and that stress enhanced MMP-9 production in vitro. The role of TAMs is to promote an inflammatory tumor environment, encouraging ovarian cancer cells to grow. Thus, biobehavioral factors are associated with both stromal and tumor expression of factors supporting angiogenesis and invasion in the tumor microenvironment in patients with ovarian cancer. Davis et al. [17] also investigated patients with ovarian cancer and showed that eudaimonic wellbeing is associated with lower tumor norepinephrine (NE) levels. Since NE is part of adrenergic signaling, which is implicated in tumor progression, these authors suggest that increasing eudaimonic well-being may improve both psychological and physiological resilience in patients with ovarian cancer.

Hoyt et al. [19] showed that a person's ability to cope with and regulate emotion affects inflammatory markers. Men who underwent radical treatment for prostate cancer and who had the ability to process their emotions (i.e. understand, make meaning of and work through these emotions) had lower expression levels of the inflammatory markers IL-6, sTNF-RII and CRP. Men (from the same patient group) who were prone to emotional expression but who were less able to process their emotional experiences had elevated levels of sTNF-RII. Thus, emotional regulation via emotional processing appeared to modulate inflammatory processes.

B. Childhood Adversity: Carli et al. [20] analyzed the correlation between the 5-HTTLPR genotype and psychological resilience and depressive symptoms in a high-risk group (male prisoners). As described above, 5-HTTLPR is the promoter region of the serotonin transporter (5-HTT) gene. The 5-HTTLPR polymorphism results in either $\mathrm{L}$ or $\mathrm{S}$ alleles. The low functional allele $(\mathrm{S})$ is associated with less efficient transcription of the 5-HTT gene [35]. Carli el al. [20] concluded that men who have been subjected to severe childhood adversities and who have the $\mathrm{L}$ allele had lower psychological resilience and increased depressive severity than men who have the S/S homozygous gene. Das et al [21] showed that a higher number of reported childhood adversities correlated with a lower resilience score. In addition, they also showed that a person who had the 7-repeat allele of the dopamine D4 receptor (DRD4) appeared to be protected against the decrease in psychological resilience associated with an increased number of childhood adversities because this decrease was only evident in persons without the 7-repeat allele.
C. War: Graham et al. [22] investigated the relationship between the 5-HTTLPR genotype and psychological resilience. This study compared resilience and the perception of community participation in war veterans that had or had not been diagnosed with traumatic brain injury. It was concluded that both, (i) the presence of traumatic brain injury and (ii) carrying the L allele were associated with lower resilience and more perceived limitations related to community participation, which is similar to the results by Carli et al. [20]. Telch et al.[23] investigated soldiers exposed to war zone stressors to identify any correlation between developing PTSD, depression or anxiety and the 5-HTTLPR genotype. Their study showed that individuals carrying one or two of the low functional alleles (S) had more symptoms of PTSD, depression and anxiety than individuals who were homozygous for the high functioning allele, L. This result was contradictory to the results of Carli and Graham, et al. [20,22].

D. High-Impact Trauma: Two studies investigated persons that, (i) had developed PTSD (the low-resilience group) or (ii) had not developed or had recovered from PTSD (high-resilience group) after being exposed to high-impact trauma (e.g., sexual assault or rape, nonsexual assault, childhood physical/sexual abuse, adult physical abuse, lifethreating accident, witnessing violent death, domestic violence, etc.). Cowdin et al. [24] showed that persons in the high-resilience group had right frontal theta activity during REM sleep. In contrast, persons in the low-resilience group lacked this activity. This result suggests that right frontal theta activity during REM sleep is a biomarker for the capacity to adaptively process emotional memory among trauma-exposed individuals. Gill et al. [25] compared women in two groups, similar to the groups above, as well as a healthy control group (women not exposed to trauma and no PTSD diagnosis). They found that the recovered group and the control group had similarly high levels of HRQOL and similarly low levels of the inflammatory markers CRP and IL-6 compared to the women who currently had PTSD. Thus, psychological recovery from PTSD was found to be associated with normal levels of both inflammatory biomarkers and HRQoL [25].

E. Stress: Three studies have analyzed the relationship between biological markers and psychological hardiness (later termed resilience), socioenvironmental conditions and oxidative damage in individuals exposed to different kinds of stress, e.g., acutely stressful situations [26,27] and chronic stress [28]. Psychological hardiness refers to three interrelated personality characteristics [36,37]: commitment (the tendency to involve oneself in, rather than experience alienation from, whatever one encounters in life), control (the tendency to think, feel and act as if one is influential, rather than helpless, in the face of the varied challenges of life) and challenge (the tendency to believe that change rather than stability is normal in life and that changes are interesting incentives to growth rather than threats to security). Sandvik et al. [27] studied navy cadets in a highly stressful military field exercise. All cadets scored high on hardiness, but some 
scored high only on commitment and control and scored low on challenge. Individuals who scored low on challenge were more stress reactive and showed a suppression of the proinflammatory marker IL-12, increased levels of the anti-inflammatory markers IL-4 and IL-10 and lower neuropeptide-Y levels. IL-12 is involved in the differentiation of naive $\mathrm{T}$ cells into Th1 cells, stimulates the production of IFN- $\gamma$ and TNF- $\alpha$ and reduces IL- 4 mediated suppression of IFN- $\gamma$. Thus, being more balanced in one's hardiness profile (i.e., having profoundly high scores in commitment, control and challenge) is linked to more moderate and healthier immuneand neuroendocrine responses to stress [27].

Previous studies have identified a polymorphism in the IL-6 promoter that interacts with adverse socioenvironmental (SE) conditions (IL-6 x SE) to promote chronic inflammation in older adults. In this study, Cole et al. [26] showed that genetic polymorphisms that interact with adverse SE conditions to increase health risk late in life may provide biological resilience against adversity earlier in life. This was shown in adolescents with low socioeconomic status (one of the most robust SE health risk factors), and a protective effect of this IL-6 polymorphism was identified. However, interpersonal stress later in adulthood affected this IL-6 $x$ SE interaction and was related to inflammatory processes. Cole et al. [26] concluded that the polymorphism in the IL-6 promoter was related to psychological resilience but also conferred a genetic vulnerability, depending on the somatic environment. Oxidative damage seems to be an important mediator of this process. Aschbacher et al. [28] showed that women under chronic stress (i.e., who had spouses with dementia) had higher levels of 8-oxoG, reflecting oxidative damage to RNA, than the control group. These results support the emerging model that chronic psychological stress exposure promotes oxidative damage through the frequent and sustained activation of the hypothalamic-pituitary-adrenal axis.

\section{F. Psychological Resilience in a Non-Trauma Setting:} One's level of psychological resilience is thought to be an important factor for successful aging. 0 Hara et al. [30] analyzed the genetic variant of the promoter region of the serotonin transporter gene and were unable to identify any significant association between the individuals carrying the S gene and psychological resilience in older adults ( $>55$ years). However, they found that patients with the $\mathrm{S}$ allele had worse cognition and lower self-reported ratings of successful aging. The researchers suggested that the $\mathrm{S}$ allele status is a biomarker for poorer self-rated success in aging and worse cognitive performance in older adults. Rana et al. [29] performed a single-nucleotide polymorphism (SNP) analysis of 65 candidate genes associated with optimism and psychological resilience in older adults ( $>50$ years). They found no significant association between individual SNPs and optimism or psychological resilience in a single-locus analysis. However, in multilocus polygenic analyses, associations between optimism and SNPs in the MAO-A, IL-10, and fibrinogen $\mathrm{G}$ ( $\mathrm{FGG}$ ) genes were identified, in addition to associations between psychological resilience and an SNP in the MAO-Agene [29]. Petros et al. [31] analyzed the relationship between psychological parameters and salivary cortisol and dehydroepiandrosterone sulfate (DHEA-S) levels as putative biomarkers for psychological resilience in a cross-sectional sample cohort. This group showed that psychological resilience correlated negatively with selfrated depressive symptoms, trait anxiety and early life stress and correlated positively with self-efficacy, optimism social support and well-being. Psychological resilience and DHEA-S concentrations correlated significantly. It was concluded that psychological resilience is associated with positive aspects of psychological health and salivary DHEA-S, suggesting salivary DHEA-S as a biomarker for psychologic resilience [31] Stein et al. [32] analyzed the correlation between psychological resilience and genetic variants of the promoter region of the serotonin transporter gene, 5-HTTLPR, in college students. The results showed that a higher copy number of the $\mathrm{S}$ allele in the promoter region was associated with lower psychological resilience than being homozygous for the $\mathrm{L}$ allele. This is in line with findings by Telch et al. [23] but is contradictory to the results by Carli, Graham et al. [20,22]

\section{Discussion}

The main objective of this report was to provide a scoping review of the relationship between psychological resilience and biomolecular processes in a cancer context to advise research on the interaction between psychological resilience and biomolecular variables during demanding life situations such as cancer [38]. However, only few references were identified to fit those criteria; thus, the scope was broadened to include threats other than cancer, resulting in a total of 16 peer reviewed articles. This lack of cancerrelated research shows that there is a gap in the current literature connecting the body and mind in a cancer context. In addition, a spectrum of biomolecular parameters was assessed throughout the studies, and there is no systematic method for the setup of a study coupling psychological resilience to biomolecular processes. However, there are indications that psychological resilience relates to the outcome of various diseases and that it might influence cancer diagnosis and treatment outcomes [17-19]. This result is also shown by the enhanced effect that stress, which is related to psychological resilience, has on the metastatic potential of tumor cells [15]. Taken together, these results show a prerequisite for more research to pave the way for a novel avenue of therapeutic interventions, both medical and psychosocial, for cancer patients. Biomolecular variables related to inflammation and to plasticity genes has got attention.

\section{Inflammation}

Some studies Table 1 indicate that biobehavioral factors are associated with inflammatory markers, as well as the expression of factors in the stroma and tumor that support angiogenesis and invasion in the tumor microenvironment [18]. Increased levels of both depression and stress have been shown to correlate with 
increased MMP-9 secretion by TAMs in patients with ovarian cancer. These TAMs promote an inflammatory tumor environment that is beneficial for tumor development [18], indicating that stress is significantly associated with tumor progression. Interestingly, patients with access to supportive social relationships had lower levels of these TAMs, indicating the importance of social support in cases of trauma. Eudaimonic wellbeing (happiness that is related to striving) is associated with lower tumor NE in ovarian cancer patients; thus, since adrenergic signaling is implicated in tumor progression, increasing eudaimonic wellbeing may improve both psychological and physiological resilience in patients with cancer [17].

Furthermore, additional studies strengthen the view that there is a correlation between biobehavioral factors and inflammation; although, thus far, this result has not been shown in a cancer context. It has been shown that (i) a person with an inflammatory signature (decreased levels of the proinflammatory marker IL12 and increased levels of the anti-inflammatory markers IL-4 and IL-10) is more stress reactive [27], (ii) a less inflammatory microenvironment (e.g., a decreased levels of TNF-RII, IL-6 and CRP) correlated with a better ability to process emotions [19], and (iii) persons that either recovered from PTSD or never developed PTSD after experiencing trauma have a lower inflammatory signature (lower levels of CRP and IL-6) than persons with PTSD and, in addition, reported higher levels of HRQOL [25]. Thus this finding indicates a connection between psychological behaviors and inflammation, cancer progression and prognosis. Understanding, assigning meaning to and working through emotional experiences may be a promising target of intervention to reduce inflammation with potential effects on psychological resilience and cancer outcomes.

\section{Plasticity Genes}

Another finding was that the $\mathrm{G} \times \mathrm{E}$ interaction may relate to psychological resilience. Belsky and Pluess [9] proposed that genes that had earlier been described as vulnerability genes may be more accurately described as plasticity genes, meaning that individuals - due to their genetic make-up - can be more (or less) susceptible to environmental influences. This susceptibility goes both ways. A certain genetic variation can be associated with beneficial effects in nurturing environments, while the same variation can be associated with negative effects in poor environments. A biomarker that has been investigated in several of the included studies is the genetic variant of the promoter region (5-HTTLPR) of the serotonin transporter (5-HTT) gene. However, the results are contradictory. Three studies $[23,30,32]$ showed that carrying the $S$ allele in the promotor gene is associated with a more vulnerable profile. However, two studies $[20,22]$ showed that the L allele is associated with a more vulnerable psychological profile. Carli et al. [20] showed that the genotype alone did not influence resilience and depression severity; however, among individuals exposed to high levels of childhood trauma, carriers of the L-allele had lower resilience scores and more severe depressive symptoms than those who were $\mathrm{s} / \mathrm{s}$ homozygous. This contrasts with evidence supporting the $\mathrm{S}$ allele as conferring a higher risk for psychopathology in subjects exposed to recent stressful events, again demonstrating the complexed relation between biomarkers and resilience. Another biomarker of interest is the dopamine receptor gene D4. Das et al. [21] found that the 7-repeat allele of DRD4 was associated with higher resilience scores in adults. Individuals without the 7-repeat allele showed lower resilience levels. The protective effect of the 7-repeat allele was only present in individuals who had experienced negative events during childhood. Hence, their findings are coherent with findings by Carli, et al. [20].

Taken together, these findings illustrate the complexity of $\mathrm{G} \times \mathrm{E}$ interactions. Early life experiences may cause lasting epigenetic changes and may modify gene expression, both of which, consequently, affect individual responses to environmental stressors during adulthood. Like Belsky and Pluess [9], Kim-Cohen and Turkewitz et al. [39] argue that these genes might predict psychological resilience to adversity, as well as psychopathology. Sexspecific effects; differences in the type, time and duration of stressors; and the specific composition of the sample may explain the discrepancies.

\section{Psychological Resilience Construct}

The two core concepts adversity and positive adaptation showed great variation (Table 2). In terms of adversity the studies included major, life-threatening events, such as being diagnosed with cancer; expected life-changes, such as growing old; and chronic stressors, such as caring for a sick spouse. Additionally, studies varied in terms of when in time participants had been exposed to adversity. In studies investigating childhood trauma exposure, adversity had occurred years before the biomolecular data were collected $[24,25]$. In other studies, the data were collected closer in time to the stressful event. For example, Sandvik et al. [27] collected blood samples five days after a demanding military exercise. Previous reviews [2,4] have emphasized that the definition of positive adaptation must be appropriate for the type of adversity. In the context of serious life-threatening events, adaptation may be defined as fewer psychopathological symptoms and/or not meeting the criteria for a major depression or PTSD. Several articles included in the present review took this approach to adaptation, e.g. Cowdin et al. [24]. Regarding everyday stressors, adaptation may instead be defined as continued optimism and/or sustained HRQOL [29,31]. Some articles combined these approaches, hence using both psychiatric illnesses (i.e., the absence of symptoms) and sustained life quality as signs of positive adaptation. Several studies measured psychological resilience directly, in most cases using the CD-RISC instrument. Instead of measuring symptoms of psychopathology or particular personal factors such as self-efficacy or optimism, the CD-RISC more comprehensively measures both intrapsychic and contextual factors. It rests on the conceptualization of psychological resilience as a protective process to employ in 
response to adverse conditions. In comparison, articles screening for psychopathologies investigate the "result" of psychological resilience, using methods such as depression or PTSD inventories. Thus, applying the construct of psychological resilience varied in the studies included.

This scoping review aimed to examine the existing research regarding the association between psychological resilience and biomolecular variables. The data collection strategy rested on the stages outlined by Arksey and O'Malley [16]. It included searches mainly via electronic databases and reference lists. Some practical limits had to be placed on the searches. Only papers in English was included and it cannot be ruled out that relevant studies therefore were excluded. Second, the search strategy included the term "psychological resilience". Since there are terms related to psychological resilience (e.g., sense of coherence, hardiness, positive adaptation, etc.) not used as keywords, relevant references may have been overlooked. Finally, this scoping review does not appraise the quality of the research evidence, leading to a large range of study designs and methods being included - a methodological limitation also described by Arksey and O'Malley [16].

\section{Conclusion}

In conclusion, this scoping review demonstrates that only very few reports have explored an association between psychological resilience and biomarkers in cancer, hence no firm conclusion can be drawn. Furthermore, the associations reported is only based on a few single biomarkers involved in inflammation or variations in a promotor region, where also the results are partly contradictory. Since individual protein/genetic biomarkers contains too little information to form the basis for any clinical conclusions, there is a need to focus on connection macroscopic events, such as metastatic ability [15] with massive screening of biomarkers. Also the approach to assessing psychological resilience needs to be stringent in terms of adversity and adaptation. Such approaches will allow, for the first time, to analyze massive amount of biological data and then to start building biomarker networks, genetic maps and signaling pathways that will advance the knowledge of the association between psychological resilience and biomolecular variables.

\section{Acknowledgment}

This study was supported by funding from CREATE Health Cancer Center, Lund University. We acknowledge the SCAN-B Resilience study group.

\section{Conflict of interest}

Ulrika Axelsson, Johanna Månsson, Per Johnsson, Carl Borrebaeck, Lisa Rydén, Patrik Edén and Ingalill Rahm Hallberg declare that there is no conflict of interest.

\section{Funding}

This research received no specific grant from any funding agency in the public, commercial, or not-for- profit sectors.

\section{Note}

We have been in contact with PROSPERO and today one cannot register Scoping reviews in PROSPERO, why this is not done. As described in "A scoping review of scoping reviews: advancing the approach and enhancing the consistency. Mai T. Pham et al. There is, as of today, no established checklist to follow for Scoping reviews. However we follow the same method (Arksey H, O'Malley L. Scoping studies: towards a methodological framework. International Journal of Social Research Methodology. 2005;8(1):19-32.) as Pham et al do. The methodological framework described by Arksey and O'Malley consists of five stages: (i) Identifying the research question; (ii) Identifying relevant studies; (iii) Study selection; (iv) Charting the data and (v) Summarizing and reporting the results.

\section{References}

1. Masten AS (2011) Resilience in children threatened by extreme adversity: Frameworks for research, practice, and translational synergy. Dev Psychopathol 23(2): 493-506.

2. Fletcher D, Sarkar M (2013) Psychological resilience, a review and critique of definitions, concepts, and theory. European Psychologist 18(1): 12-23.

3. Herrman H, Stewart DE, Diaz Granados N (2011) What is resilience? Can J Psychiatry 56(5): 258-265.

4. Luthar SS, Cicchetti D, Becker B (2000) The construct of resilience: A critical evaluation and guidelines for future work. Child Dev 71(3): 543562 .

5. Bowes L, Jaffee SR (2013) Biology, genes, and resilience: Toward a multidisciplinary approach. Trauma Violence Abuse 14(3): 195-208.

6. Kim Cohen J, Moffitt TE, Caspi A (2004) Genetic and environmental processes in young children's resilience and vulnerability to socioeconomic deprivation. Child Dev 75(3): 651-668.

7. Kim Cohen J, Gold AL (2009) Measured gene-environment interactions and mechanisms promoting resilient development. Current Directions in Psychological Science 18(3): 138-142.

8. Kim Cohen J, Caspi A, Taylor A, Williams B, Newcombe R, et al. (2006) Maoa, maltreatment and gene-environment interaction predicting children's mental health: New evidence and a meta-analysis. Molecular Psychiatry 11(10): 903-913.

9. Belsky J, Pluess M (2009) The nature (and nurture?) of plasticity in early human development. Perspect Psychol Sci 4(4): 345-351.

10. Cal SF, Sá LRd, Glustak ME (2015) Resilience in chronic diseases: A systematic review Cogent. Psychology 2(1).

11. Chida Y, Hamer M, Wardle J, Steptoe A (2008) Do stress-related psychosocial factors contribute to cancer incidence and survival? Nature Clinical Practice Oncology 5(8): 466-475.

12. Pinquart M, Duberstein PR (2010) Depression and cancer mortality: A meta-analysis. Psychol Med 40(11): 1797-1810.

13. Pinquart M, Duberstein PR (2010) Associations of social networks with cancer mortality: A meta-analysis. Critical Reviews in Oncology Hematology 75(2): 122-137.

14. Lutgendorf SK, Andersen BL (2015) Biobehavioral approaches to cancer progression and survival: Mechanisms and interventions. Am Psychol 70(2): 186-197.

15. Creed SJ, Le CP, Hassan M, Pon CK, Albold S, et al. (2015) Beta2adrenoceptor signaling regulates invadopodia formation to enhance 
tumor cell invasion. Breast Cancer Res 17(1): 145.

16. Arksey H, O Malley L (2005) Scoping studies: Towards a methodological framework. International Journal of Social Research Methodology 8(1): 19-32.

17. Davis LZ, Slavich GM, Thaker PH, Goodheart MJ, Bender DP, et al. (2015) Eudaimonic well-being and tumor norepinephrine in patients with epithelial ovarian cancer. Cancer 121(19): 3543-3550.

18. Lutgendorf SK, Lamkin DM, Jennings NB, Arevalo JM, Penedo F, et al. (2008) Biobehavioral influences on matrix metalloproteinase expression in ovarian carcinoma. Clin Cancer Res 14(21): 6839-6846.

19. Hoyt MA, Stanton AL, Bower JE, Thomas KS, Litwin MS, et al. (2013) Inflammatory biomarkers and emotional approach coping in men with prostate cancer. Brain, Behavior, and Immunity 32: 173-179.

20. Carli V, Mandelli L, Zaninotto L, Roy A, Recchia L, et al. (2011) A protective genetic variant for adverse environments? The role of childhood traumas and serotonin transporter gene on resilience and depressive severity in a high-risk population. Eur Psychiatry. 26(8): 471-478.

21. Das D, Cherbuin N, Tan X, Anstey KJ, Easteal S, et al. (2011) Drd4exoniii-vntr moderates the effect of childhood adversities on emotional resilience in young-adults. PLoS One 6(5): e20177.

22. Graham DP, Helmer DA, Harding MJ, Kosten TR, Petersen NJ, et al. (2013) Serotonin transporter genotype and mild traumatic brain injury independently influence resilience and perception of limitations in veterans. J Psychiatr Res 47(6): 835-842.

23. Telch MJ, Beevers CG, Rosenfield D, Lee HJ, Reijntjes A, et al. (2015) 5-httlpr genotype potentiates the effects of war zone stressors on the emergence of ptsd, depressive and anxiety symptoms in soldiers deployed to iraq. World Psychiatry 14(2): 198-206.

24. Cowdin N, Kobayashi I, Mellman TA (2014) Theta frequency activity during rapid eye movement (rem) sleep is greater in people with resilience versus ptsd. Experimental Brain Research 232(5): 1479-1485.

25. Gill JM, Saligan L, Lee H, Rotolo S, Szanton S, et al. (2013) Women in recovery from ptsd have similar inflammation and quality of life as nontraumatized controls. Journal of Psychosomatic Research 74(4): 301306.

26. Cole SW, Arevalo JM, Manu K, Telzer EH, Kiang L, et al. (2011) Antagonistic pleiotropy at the human il6 promoter confers genetic resilience to the pro-inflammatory effects of adverse social conditions in adolescence. Dev Psychol 47(4): 1173-1180.

27. Sandvik AM, Bartone PT, Hystad SW, Phillips TM, Thayer JF, et al. (2013)

\section{ISSN: 2574-1241}

DOI: 10.26717/BJSTR.2019.23.003960

Ulrika Axelsson. Biomed J Sci \& Tech Res

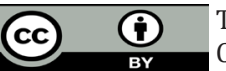

This work is licensed under Creative

Commons Attribution 4.0 License

Submission Link: https://biomedres.us/submit-manuscript.php
Psychological hardiness predicts neuroimmunologica responses to stress Psychol Health Med 18(6): 705-713.

28. Aschbacher K, O Donovan A, Wolkowitz OM, Dhabhar FS, Su Y, et al. (2013) Good stress, bad stress and oxidative stress: Insights from anticipatory cortisol reactivity Psychoneuroendocrinology 38(9): 16981708.

29. Rana BK, Darst BF, Bloss C, Shih PA, Depp Cet al. (2014) Candidate snp associations of optimism and resilience in older adults: Exploratory study of 935 community-dwelling adults. Am J Geriatr Psychiatry 22(10): 997-1006.

30. 0’Hara R, Marcus P, Thompson WK, Flournoy J, Vahia I, et al. (2012) 5 -httlpr short allele, resilience, and successful aging in older adults. The American Journal of Geriatric Psychiatry 20(5): 452-456.

31. Petros N, Opacka Juffry J, Huber JH (2013) Psychometric and neurobiological assessment of resilience in a non-clinical sample of adults. Psychoneuroendocrinology 38: 2099-2108.

32. Stein MB, Campbell Sills L, Gelernter J (2009) Genetic variation in 5httlpr is associated with emotional resilience Am J Med Genet B Neuropsychiatr Genet 150B (7): 900-906.

33. Connor KM, Davidson JR (2003) Development of a new resilience scale: The connor-davidson resilience scale (cd-risc). Depress Anxiety 18(2): 76-82.

34. Bartone PT (2007) Test-retest reliability of the dispositional resilience scale-15, a brief hardiness scale. Psychol Rep 101(3): 943-944.

35. Lesch KP, Bengel D, Heils A, Sabol SZ, Greenberg BD, et al. (1996) Association of anxiety-related traits with a polymorphism in the serotonin transporter gene regulatory region. Science 274(5292): 15271531.

36. Kobasa SC (1979) Stressful life events, personality, and health: An inquiry into hardiness. J Pers Soc Psychol 37(1): 1-11.

37. Kobasa SC, Maddi SR, Kahn S (1982) Hardiness and health: A prospective study. J Pers Soc Psychol 42(1): 168-177.

38. Axelsson U, Ryden L, Johnsson P, Edén P, Månsson J et al. (2018) A multicenter study investigating the molecular fingerprint of psychological resilience in breast cancer patients: Study protocol of the scan-b resilience study. BMC Cancer 18(1):789.

39. Kim Cohen J, Turkewitz R (2012) Resilience and measured geneenvironment interactions. Dev Psychopathol 24(4): 1297-306.

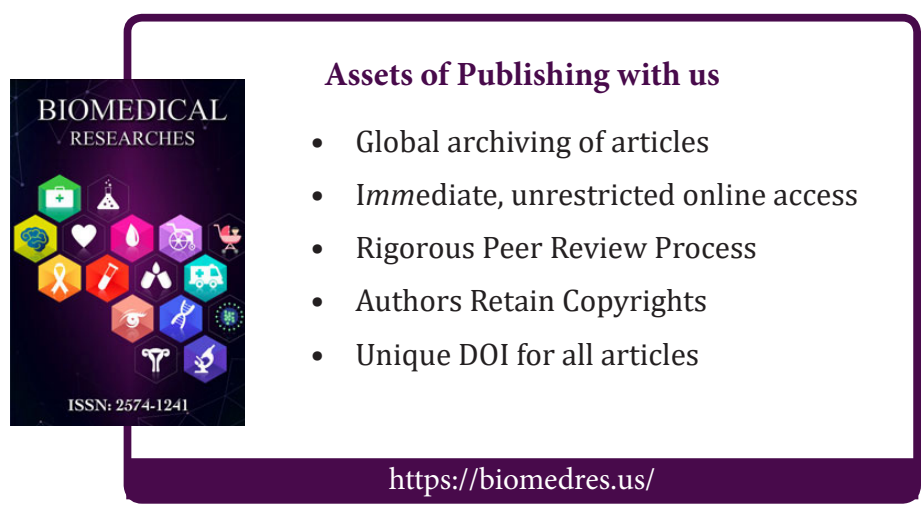

Copyright@ Ulrika Axelsson | Biomed J Sci \& Tech Res | BJSTR. MS.ID.003960. 